\title{
Laser beam profiling: experimental study of its influence on single-track formation by selective laser melting
}

\author{
Ivan V. Zhirnov, Pavel A. Podrabinnik, Anna A. Okunkova a and Andrey V. Gusarov \\ Moscow State University of Technology "STANKIN", 1 Vadkovsky per., 127994 Moscow GSP-4, Russian Federation
}

Received 24 September 2015, Accepted 2 October 2015

\begin{abstract}
In the article the experimental study of influences of laser beam profiling on the microstructure of the material obtained by selective laser melting is presented. Microstructure was researched by the example of single-track formation. For these needs the optical and video-monitoring stand was constructed. The defects of single-track formation were obtained by microscopy and video monitoring. The technological gaps for each laser beam profile give the possibility to use higher power for laser systems in the future with the purpose to improve productivity of SLM processing.
\end{abstract}

Key words: Additive manufacturing / laser beam modulation / optical monitoring and diagnosis

\section{Introduction}

At present fast prototyping methods are developing rapidly. One of the prospective methods allowing manufacturing of complex geometry objects in a short time from wide range of materials is selective laser melting $[1-3]$. This method has found wide use in different branches of the industry such as machine building, aircraft construction and also health care where there is need of production of individual prostheses and implants.

Principle of this method is in powder layer-by-layer melting by a laser beam in accordance with an initial 3D model: powder material is applied at the substrate and flattened with a roller or scraper and melted by a laser beam in accordance with current cross-section of the 3D model. The substrate is lowered one level below and the process is repeated. The future object is formed in a powder material layer; so minimized numbers of supports are required for article defect-free formation $[4,5]$.

However, there are some negative process effects, which negatively influence on the final quality of the objects. The process tasks to reduce them have not been solved still. In general these effects are associated with the powder material granules' melting process.

Observation of selective laser melting has shown [6] that at the melting moment some powder material granule jetting has occurred under the effect of the laser beam in the working area. This results in "scattering" of powder granules, which damages the granulometric composition near the working area of each layer. It results in

${ }^{a}$ Corresponding author: a.okunkova@stankin.ru a series of negative effects such as chemical interaction of powder material granules with protective atmosphere in the chamber. This happens because of overheating in the middle of the molten pool. This creates a high speed stream of liquid metal in the working area; as well the energy loses on vaporization of the liquid metal, Marangoni effect, etc. [7].

Described negative effects accompanying the selective laser melting process may be directly associated with the laser beam power density distribution. Wide spread kind of laser beam power density distribution in the laser sources of the SLM-machines gets the name "Gauss" distribution or $\mathrm{TEM}_{00}$ mode. As expected this problem and its effects may be reduced by means of laser beam modification. The means includes the optical system for laser beam modulation and the system of optical monitoring. So the power density gradient may be corrected with the purpose to create the optimal thermal- and mass-transfer conditions at the molten pool surface, liquidating and reducing the powder material granule jetting effect [8-10].

Other aspect of work for improvement of the object quality is the research for process parameters. The criterion of their definition is a correspondence to the required parameters of the objects including the geometric accuracy, the quality of microstructure and mechanical properties. Laser beam powder melting process encloses a set of inconstant input parameters and as a consequence their combinations. This can significantly complicate the research of optimal parameters of SLM-processing. Due to this reason it is necessary to install the means of optical monitoring system of SLM-processing. The system 
allows collecting of enough data for diagnosis of negative effects and also an explanation of some processes [11].

Now there is no melting process control system at industrial machines [12]. The quality of 3D objects' production by SLM is controlled approximately, just by watching it during processing. The task of development of optical monitoring means for SLM processing including the laser beam modulation system is a matter of topical interest nowadays and permits to improve end product quality.

\section{Problem statement}

Previous studies in the field of laser beam modulation have shown the possibility of obtaining uniform temperature fields in the heating zone with the use of Flat-top and Inverse Gaussian laser beam power density distribution [13]. Many scientists in their papers have mentioned nearly the same set of SLM process parameters including twelve positions associated with laser beam, gas atmosphere and powder parameters $[14,15]$.

All SLM process parameters are divided at the input and output parameters. Main input controlled parameters may comprise laser beam power, laser wave length, pulsing mode, beam focus diameter, beam profile, beam quality factor, scanning speed, and used laser beam processing strategy. Output parameters of the SLM-process are considered as some measured parameters, which influence on the functionality of the final product: obtained surface geometric accuracy surface roughness, obtained microstructure (presence of pores, residual cracks) and main mechanical properties. The measured output parameters of the end product are in correlation with the measured parameters of the object' single track as: single track geometric parameters (height, width, depth), its metallurgical and geometric uniformity and powder consolidation zone (heat affected zone). Besides SLM process input controlled parameters there are non-controlled or partially controlled parameters of protective atmosphere in the chamber of the SLM-machine and powder material parameters. Normally the atmosphere properties depend on its composition and thermal properties as well as atmosphere' and substrate' temperatures and chamber' air tightness (presence of oxygen). The chemical composition of the powder alloy, its thermal properties (not every powder is suitable for SLM processing), powder granules' characteristics (shape, size; the most preferable shape of the granules for SLM processing is round, which is more suitable for energy absorption) and the thickness of the powder layer, which depends on the granules' size, can be defined as the main powder characteristics.

So the main objectives of this study have been formulated: to analyze existing machines for selective laser melting; to develop the optical system for laser beam modulation and monitoring and integrate it into the experimental stand; to define the system parameters for the alternative laser beam power density distributions; to test the developed system by optical monitoring and diagnosis assets.

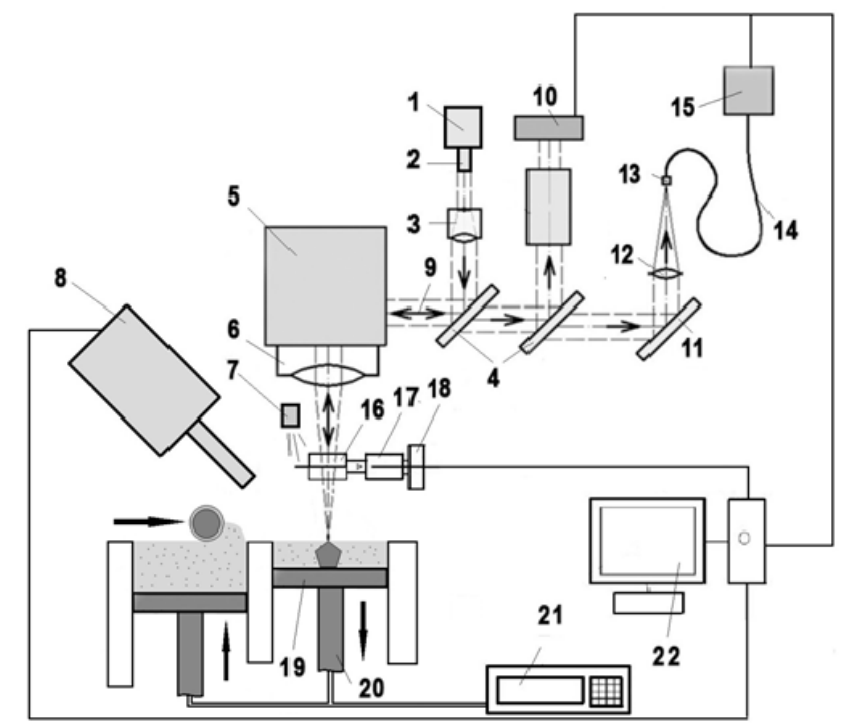

Fig. 1. Scheme of the developed experimental stand: 1 - laser beam source; 2 - collimator; 3 - pi-Shaper; 4 - mirror; 5 scanator; 6 - F-theta lens; 7 - laser illumination; 8 - high speed CCD camera; 9 - laser beam travel path; 10 - short range IRcamera; 11 - mirror; 12 - pyrometer lens; 13 - signal receiver; 14 - optic fiber; 15 - pyrometer; 16 - BCube optic mirror; 17 attenuator; 18 - LaserCam-HRTM CCD camera; 19 - working platform; 20 - moving piston; 21 -electronic micrometer; 22 computer.

\section{Experiment}

\subsection{Development of experimental optic diagnosis stand}

At present there are several main manufacturers of selective laser melting machines including EOS (Germany), Concept (Germany), Phoenix Systems (France), Renishaw (UK). In principle all machines have the same construction: laser beam leaves collimator, comes to scanning system and then process a required pattern of powder layer according to the current program. All machines use the Gaussian laser beam distribution and as a result they have indicated the above defects, which are common to this distribution.

The experimental stand (Fig. 1) has been developed to perform studies in the laboratory of MSTU "STANKIN". The main principles of the construction of the experimental stand were performed in accordance to the typical construction of an SLM-machine.

Development of the experimental stand includes two main steps. The first step comprises the installation of an optical module assembly for laser beam modulation, its displacement and also equipment for optical monitoring and diagnosis. The second step has been aimed at provision of translational displacement for the mechanical parts of the stand as the powder bunker, the working platform with the powder layer. All these assemblies have been connected with an automatic controller to ensure high accuracy movement. 
Fiber optics ytterbium laser (LK-200-B, IPG) (1) with wave length $\lambda=1070 \mathrm{~nm}$ and maximum power $P=200 \mathrm{~W}$ has been used as laser beam source The scanator (5) which has two intercrossing mirrors, was integrated in the optical system of the stand to provide the movement of the laser beam in the working area in accordance to the program. A Pishaper (3) is installed between scanator and the laser beam source. Main purpose of this module is the transformation of the laser beam from Gaussian distribution to an alternative one using the principle of its refraction by aspherical optic surfaces. After the scanator beam passes via focusing f-theta lens (6), used for magnifying of marked area at laser processing. Then the laser beam comes directly at the working surface for processing of the powder layer. System comprising BCube optic mirror (16), attenuator (17) and LaserCamHRTM CCD-camera (18) is used for monitoring of laser beam profile. Signal is recorded directly at CCD camera. However device technical characteristics allow processing of laser with maximum power $4 \mathrm{~W}$. Therefore it is required to use the attenuator, which is a passive device reducing passing signal to required value before its sending to the camera. Signal from camera is transmitted to computer 22 where it is processed by special software and presented to operator in a form suitable for interpretation. The first element of optical monitoring and diagnosis system is a high speed CCD camera (8). It records SLM process in visible range. Maximum shooting speed is $775000 \mathrm{fps}$, maximum resolution is $1024 \times 1024$ pixels. Cavilux laser illuminator (7) to illuminate shooting area is required for operation of the camera. The camera is equipped with a light filter protecting the obtained image from radiation generated by the illuminator and also a macro object glass capable to shoot fine details of process at high-power magnification. Short range IR camera (10) is required to obtain information about the heat affected zone in process of treatment and has a resolution of $560 \times 760$ pixels, holding time is $3 \mathrm{~ms}$. Device has been calibrated with black body at 1200-1800 K temperature range. A two-wave pyrometer (15) has been used to measure temperatures during laser beam processing. It is capable to record temperatures in the range of 1200 $2900 \mathrm{~K}$.

The mechanical part of the experimental stand consists of a high accuracy low-carbon steel working platform (19), where the object is produced. It is located at piston (20), driven by a step engine, which is controlled by an electronic micrometer (21).

\subsection{Performance of experiment}

Cobalt-chromium alloy has been selected as material for this experiment. Powder has been obtained by gas atomization, has a spherical shape of particles with average diameter less than $50 \mu \mathrm{m}$. Due to space limits and high quantity of equipment required for the experiment the melting process has been performed in air without protective atmosphere. Practice of use of this material in SLM process has shown that cobalt-chromium alloy has maximum heat resistivity in comparison with other traditionally used materials as Ti6Al4V, maraging steel etc.

Powder layer thickness has been controlled with an Olympus BX51M optical microscope. An optical microscope has been used also for studying the track penetration and microstructure. Samples have been prepared at ATM Machine Tools sampling equipment (Germany). This equipment includes a cutoff machine, a hydraulic press and a polishing machine.

Powder material layer nominal thickness in this study has been $80 \mu \mathrm{m}$ and controlled by an optical microscope. According to scanator operation manual requirements substrate with powder at experimental stand has been installed on the working platform in such way to have a distance $420 \mathrm{~mm}$ from the lens to the working surface. Such distance ensures the position laser beam focus directly at the powder layer. This substrate position is considered as zero one.

The piShaper laser beam profiling system turning ring has been locked because of the experimental stand characteristics and with the purpose to reduce the influence of possible errors. The modulation of laser beam power density distribution has been performed by changes of this distance, because laser beam power density distribution depends on the distance between scanator lens focus and working surface. The effective diameter and effective area have been determined and measured as $0.111 \mathrm{~mm}$ and $0.261 \mathrm{~mm}^{2}$ correspondingly for the obtained laser beam profile.

For calculation of the distance to obtain different laser beam power density distribution the following formula has been used (1):

$$
R=\frac{8 \times \lambda \times f^{\prime 2}}{\pi \times D^{2}}
$$

where $\lambda$ is laser wave length, $f^{\prime}$ is $F$-theta lens focus distance, $D$ is collimator outlet laser beam diameter.

With the distance between different distributions it is possible to move the working surface so to perform melting of metal powder at different laser beam power density distribution. Test single track melting has been done on the empty substrate. Comparison of obtained track thickness with laser beam effective diameter at different distributions permits to evaluate achievement of one or other mode at this level.

The working surface has been moved in the following way:

- working surface position has been considered as absolute zero when it is at the same level with experimental stand working platform. This position corresponded to Flat-top distribution,

- the Gaussian distribution was obtained in the position- $15 \mathrm{~mm}$ relative to the zero mark in the focus of $F$-theta lens

- position-5 mm corresponded to Flat-top distribution.

Using LaserCam-HRTM CCD camera laser beam effective diameter and area have been determined for Flat-top and Inverse Gaussian distributions (Tab. 2). 
I.V. Zhirnov et al.: Mechanics \& Industry 16, 709 (2015)

Table 1. CoCrMo powder chemical composition.

\begin{tabular}{ccccccccc}
\hline Element & Co & Cr & Mo & Si & Mn & Fe & C & Ni \\
\hline Content (\%) & $60-65$ & $26-30$ & $5-7$ & $>1$ & $>1$ & $>0.75$ & $>0.16$ & $>0.1$ \\
\hline
\end{tabular}

Table 2. Effective diameters and areas of power density distribution, measured with LaserCam HRTM CCD camera.

\begin{tabular}{ccc}
\hline Power density distribution & Effective diameter $(\mathrm{mm})$ & Effective area $\left(\mathrm{mm}^{2}\right)$ \\
\hline Gaussian distribution & 0.111 & 0.261 \\
Flat-top distribution & 0.260 & 0.165 \\
Inverse Gaussian distribution & 0.347 & 0.124 \\
\hline
\end{tabular}

The final object produced by SLM can be represented as a superposition of the single tracks. So it is reasonable to start the research of the optimal parameters for SLM-processing on the example of single-track formation. Experiment plans comprised elaboration of twenty-five groups of parameters, five single tracks per each group. All experiments have been performed using the developed optical diagnosis system. A high speed camera Photron SA5 with $810 \mathrm{~nm}$ filter has been used for process visualization. The filter was chosen to prevent negative effects of laser and thermal radiation. Camera parameters have been selected to provide the best recording of powder particles' ejection from the molten pool. Frame rate and pulse frequency of illumination is $1000 \mathrm{~Hz}$, shutter speed is 999 microseconds, and resolution is $1024 \times 1024$ pixels.

\section{Results}

Using obtained radiations single SLM-tracks have been processed. For each distribution two input factors have been varied: laser beam power within 10-200 W and scanning speed within $5-100 \mathrm{~mm} . \mathrm{s}^{-1}$. For all distributions two main process gaps of stable track formation have been observed (at low and high power). For Gaussian distribution it was $P=30 \ldots 70 \mathrm{~W}, V=5 \ldots 20 \mathrm{~mm} . \mathrm{s}^{-1}$ and $P=130 \ldots 150 \mathrm{~W}, V=40 \ldots 70 \mathrm{~mm} . \mathrm{s}^{-1}$; for Flat-top distribution it was $P=50 \ldots 70 \mathrm{~W}, V=5 \ldots 20 \mathrm{~mm} . \mathrm{s}^{-1}$ and $P=130 \ldots 200 \mathrm{~W}, V=40 \ldots 80 \mathrm{~mm} . \mathrm{s}^{-1}$; for Inverse Gaussian distribution it was $P=70 \ldots 130 \mathrm{~W}$, $V=5 \ldots 20 \mathrm{~mm} \cdot \mathrm{s}^{-1}$ and $P=150 \ldots 200 \mathrm{~W}, V=$ $40 \ldots 100 \mathrm{~mm}^{-1} \mathrm{~s}^{-1}$.

With the increase of the effective diameter of different laser beam profiles the increase of technological gaps and their shift to area of higher power and speed has been observed. This gives optimistic forecasts for use of high-power lasers sources for the future development of SLM-processing.

Two sets of parameters $\left(V=10 \mathrm{~mm} \cdot \mathrm{s}^{-1}, P=70 \mathrm{~W}\right.$ and $V=70 \mathrm{~mm} . \mathrm{s}^{-1}, P=170 \mathrm{~W}$ (Fig. 2)) gave a stable track formation in two technological gaps for each laser beam profile. These parameters clearly demonstrated the influence, which was obtained by modulation of the laser beam, on the obtained single tracks' structure. Meanwhile power value $200 \mathrm{~W}$ and scanning speed $100 \mathrm{~mm} . \mathrm{s}^{-1}$ are limits of technological gaps due to technical characteristics of developed experimental stand.
At low power processing with Gaussian distribution the bigger depth of penetration due to higher energy concentration in the center of the laser beam spot has been observed and this shows dagger-like nature of penetration. This effect has a negative influence on future object quality because underlying layers multiple melt and solidify. Normally the shallow penetration is enough to have a solid bond with substrate, which is obtained with Flat-top and Inverse Gaussian profiles.

The opposite effect is observed with the higher power of the laser source: depth of molten pool increases with the increase of the effective diameter. The minimal depth of penetration is observed with Gaussian laser beam profile. Due to uneven increase of scanning speed and laser beam power the heat input reduction has been observed.

However optical diagnosis has shown the presence of some dynamic component during single track formation. This is always the reason of formation of such microstructure defects as pores and cracks and tracks themselves have a wavelike nature of solidification.

Also the technological gap of stable SLM-parameters, which are obtained with the higher power for Inverse Gaussian laser beam power density distribution, has been large and limited with technical characteristics of used equipment. The experiment is planned to continue with more powerful laser source.

The data about powder particles' ejection because of laser beam thermal hit have been obtained using an optical monitoring system. This phenomenon has been analyzed for all three-power density distributions according to such parameters as particle ejection speed, their quantity and direction. Recording of SLM process with a highspeed camera demonstrates the decrease of number of granules escaping the molten pool and significantly reduce the speed of these particles by use of Inverse Gaussian power density distribution.

The reduction of the Marangoni effect, which was obtained by using optical monitoring assets, positively influenced on the microstructure of the single tracks including reduction of the defects as pores and cracks during the single tracks' formation.

\section{Conclusion}

Within this study the optical monitoring and diagnosis system for the needs of selective laser melting has been 


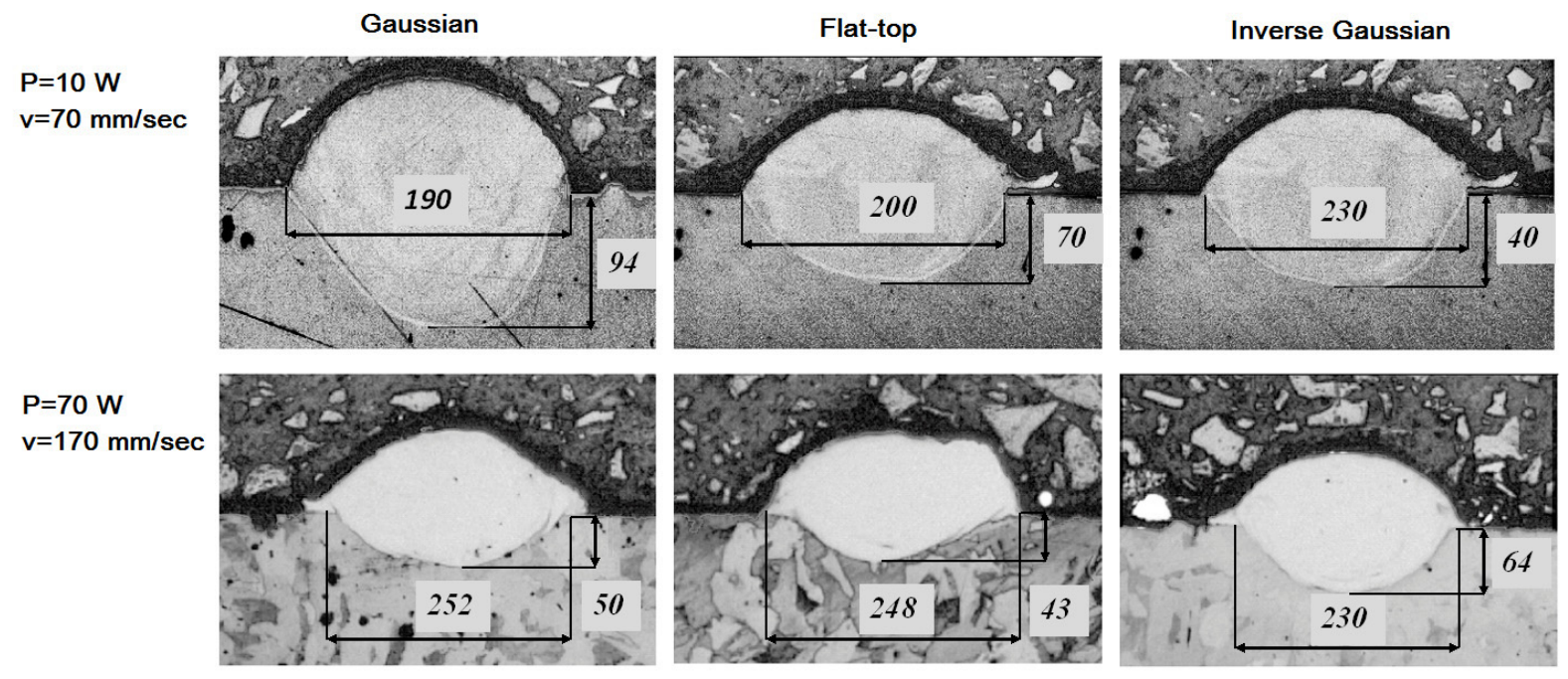

Fig. 2. Single track optic microscopy results.

developed. Set of studies of process with non-Gaussian laser beam power density distributions has been performed and obtained data have been analyzed.

In the process of the study it has been found that single tracks obtained at different power density distributions have differences both in microstructure and track geometry. So use of non-Gaussian laser beam power density distribution results in a significant reduction of the powder consolidation zone and this is a positive moment at observation of manufactured object dimensional allowances. The penetration into the substrate of the working platform for Flat-top laser beam profile has better results for single track formation. For Flat-top laser beam profile a more uniform structure of the track and better metallurgical bond with the substrate is obtained.

These phenomena permit to conclude that laser beam power density distribution has a significant influence on the selective laser melting process, in particular at higher power.

Studies in the field of selective laser melting process optical monitoring have shown that the system of video monitoring at the working area is capable to be used to evaluate integrally the melting process for indicating the defects and to control the formation of the laser path. The influences of the main input SLM parameters as scanning speed, thickness of powder layer, scanning speed on the quality of the produced single tracks and end 3D-objects obtained by using an axial diagnosis system can be evaluated in detail. This is required for implementation of new types of powder materials with composite granules' size.

R\&D tasks' solution permits to apply the system in industrial SLM-machines and perform optical monitoring of process directly during products' manufacturing.

Acknowledgements. This work has been financed by the Ministry of Education and Science of the Russian Federation in the frame of governmental task in the field of scientific activities (task order No. 9.811.2014/K). The work is carried out on the equipment of the Center of collective use of
MSTU "STANKIN" with financial support of the Ministry of Education and Science of Russian Federation, the Agreement No. 14.593.21.0004 of 04.12.2014, the unique identifier of the Project RFMEFI59314X0004.

\section{References}

[1] M. Doubenskaia, M. Pavlov, S. Grigoriev, E. Tikhonova, I. Smurov, Comprehensive optical monitoring of selective laser melting, J. Laser Micro Nanoengineering 7 (2012) 236-243

[2] M. Doubenskaia, M. Pavlov, S. Grigoriev, I. Smurov, Definition of brightness temperature and restoration of true temperature in laser cladding using infrared camera, Surf. Coat. Technol. 220 (2013) 244-247

[3] L. Esteban-Tejeda, B. Cabal, F. Malpartida, R. LópezPiriz, R. Torrecillas, E. Saiz, A.P. Tomsia, J.S. Moya, Soda-lime glass-coating containing silver nanoparticles on Ti-6Al-4V alloy, J. Eur. Ceramic Soc. 32 (2012) 27232729

[4] A.V. Gusarov, I. Yadroitsev, Ph. Bertrand, I. Smurov, Heat transfer modelling and stability analysis of selective laser melting, Appl. Surf. Sci. 254 (2007) 975-979

[5] A.V. Gusarov, I.S. Malakhova-Zjablova, A.V. Perestoronina, Calculation of Residual Stresses under Selective Laser Melting of Powders, Metallofizika I Noveishie Tekhnologii 35 (2013) 1501-1516

[6] I.V. Shishkovsky, I.A. Yadroitsev, I. Yu Smurov, Manufacturing three-dimensional nickel titanium articles using layer-by-layer laser-melting technology, Technical Phys. Lett. 39 (2013) 1081-1084

[7] I. Yadroitsev, Ph. Bertrand, I. Smurov, Parametric analysis of the selective laser melting process, Appl. Surf. Sci. 253 (2007) 8064-8069

[8] I. Yadroitsev, I. Smurov, Selective laser melting technology: from the single laser melted track stability to 3D parts of complex shape, Phys. Proc. 5 (2010) 551-560

[9] I. Yadroitsev, L. Thivillon, Ph. Bertrand, I. Smurov, Strategy of manufacturing components with designed internal structure by selective laser melting of metallic powder, Appl. Surf. Sci. 254 (2007) 980-983 
[10] I. Yadroitsev, A. Gusarov, I. Yadroitsava, I. Smurov, Single track formation in selective laser melting of metal powders, J. Mater. Process. Technol. 210 (2010) 16241631

[11] S.N. Grigoriev, V.I. Teleshevskii, Measurement problems in technological shaping processes, Measurement Techniques 54 (2011) 744-749

[12] Yu. I. Shalapko, T.V. Tarasova, Processes of dynamics of surface layers during low-amplitude fretting, J. Friction Wear 34 (2013) 166-174
[13] I. Yadroitsev, P. Bertrand, G. Antonenkova, S. Grigoriev, I. Smurov, Use of track/layer morphology to develop functional parts by selective laser melting, J. Laser Appl. 5 (2013) 052003

[14] D. Kotoban, S. Grigoriev, I. Shishkovsky, Study of 3D laser cladding for Ni85Al15 superalloy, Phys. Proc. 56 (2014) 262-268

[15] T.V. Tarasova, Prospects of the use of laser radiation for raising the wear resistance of corrosion-resistant steels, Metal Sci. Heat Treatment 52 (2010) 284-288 